\title{
Vortex matter in mesoscopic two-gap superconducting disks: Influence of Josephson and magnetic coupling
}

\author{
R. Geurts, ${ }^{*}$ M. V. Milošević, ${ }^{\dagger}$ and F. M. Peeters ${ }^{\ddagger}$ \\ Departement Fysica, Universiteit Antwerpen, Groenenborgerlaan 171, B-2020 Antwerpen, Belgium
}

(Received 4 February 2010; published 16 June 2010)

\begin{abstract}
The effects of the coupling between two electronic condensates in two-gap mesoscopic superconductors are studied within the Ginzburg-Landau theory using a finite difference technique. In applied magnetic field, we derive the dependency of the size of the vortex on the sample size and the strength of the Josephson coupling. In addition, we elaborate on the dependence of the critical temperature and field on the parameters of the coupled condensates. We demonstrate further the existence and stability of fractional states, for which the two condensates comprise different vorticity. Moreover, we also found pronounced asymmetric fractional states and we show their experimentally observable magnetic response. Finally, we introduce the magnetic coupling between condensates, and study in particular the case where one band is type II and the other is type I, i.e., the sample is effectively of I.x type. The calculated $M(H)$ loops show a clear signature of the mixed type of superconductivity, which we find to be strongly affected by the ratio of the coherence lengths in the two condensates.
\end{abstract}

DOI: 10.1103/PhysRevB.81.214514

PACS number(s): 74.78.Na, 74.25.Dw, 74.25.Uv

\section{INTRODUCTION}

$\mathrm{MgB}_{2}$ is the first superconductor unambiguously shown to possess two superconducting gaps. ${ }^{1}$ Since its discovery in $2001,{ }^{2}$ a lot of research was conducted on this specific material as well as on two-band superconductors in general. In its class of binary compounds and metallic superconductors, $\mathrm{MgB}_{2}$ turns out to have the highest critical temperature known today, $T_{c}=39 \mathrm{~K}$. Its bulk critical field is strongly anisotropic: $3.5 \mathrm{~T}$ along the $c$ axis of the crystal and $17 \mathrm{~T}$ in the $a b$ plane and can reach as high as $43 \mathrm{~T}$ in films. ${ }^{3-5}$

While the mechanism of its superconductivity is not yet entirely understood, it has been experimentally proven that $\mathrm{MgB}_{2}$ has two separate superconducting gaps. For example, in Refs. 6 and 7 the separately imaging of the $\pi$ or $\sigma$ bands was demonstrated. On the theoretical side, one considered two order parameters to describe the superconducting properties of $\mathrm{MgB}_{2}$. One of the first Ginzburg-Landau (GL) descriptions of multigap superconductors was developed by Zhitomirsky and Dao, starting from microscopic theory. ${ }^{8}$ Fitting to experimental results, the authors pinpointed the values of several GL parameters relevant for $\mathrm{MgB}_{2}$, and derived analytical expressions for the critical parameters. In Ref. 9 the same authors discussed the anisotropy of $H_{c 2}$ within the GL framework. They considered only the direct exchange of Cooper pairs between condensates, i.e., the so-called "Josephson" coupling. In the work of Askerzade et al. a different interaction between the bands was investigated-the drag effect, ${ }^{10-13}$ which is described in the GL formalism through the coupling of the gradient terms of the two condensates. The apparent agreement with experiment led these authors to fitting parameters for the GL model of $\mathrm{MgB}_{2}$.

$\mathrm{MgB}_{2}$ is generally accepted to be a type-II superconductor. However, in a very clean sample, Moshchalkov et al. estimated that one of the bands could be type-I and the other type-II. ${ }^{14,15}$ The resulting system exhibits behavior that cannot be attributed to either type, thus the name type $1.5 \mathrm{su}$ perconductivity seemed credible. Indeed, the authors found a strong clustering of vortices, a phenomenon which they ascribe to a combination of attractive and repulsive vortexvortex interaction. Actually, Ref. 16 reported a positive surface energy for vortices whenever the coherence lengths of the two bands are comparable. In Ref. 17 the semi-Meissner state was predicted theoretically for a two-gap superconductor and for superconductors which do not belong to either of the two classes type-I or type-II. The possibility of vortices carrying noninteger flux was studied in Ref. 18.

Surprisingly, virtually all studies done on two-gap superconductors (TGS) up-to-date concern bulk samples. It is known however that mesoscopic superconductivity bears a number of fascinating phenomena, ranging from specific vortex states, to enhancement of critical parameters by quantum tailoring. The only existing example of such a study is the work of Chibotaru et al. on mesoscopic disks. ${ }^{19}$ As a novelty, the authors found that fractional vortex states (when bands have different vorticity) can be realized in a TGS and can even be thermodynamically stable. However, those results turned out to be specific to the case of very weak coupling and not realistic for $\mathrm{MgB}_{2}$.

In this paper we analyze the fundamental properties and vortex matter of mesoscopic disk-shaped two-gap superconductors using the Ginzburg-Landau formalism, where the electronic exchange between condensates occurs through Josephson coupling, and magnetic exchange between condensates is allowed for. The latter mechanism has not yet been studied in detail up to now. The paper is organized as follows. In Sec. II, after describing the theoretical approach, we focus on the effects of Josephson coupling on the size of a vortex core, the unique vortex states and their $H-T$ stability regions, and the critical temperature and field as a function of coupling strength. In Sec. III, we introduce the screening of the magnetic field into the theoretical formalism, and illustrate the influence of the magnetic coupling between the two condensates on the vortex states, particularly in the case of type I.x superconductivity. Magnetic signatures of the different features are discussed in the light of potential observation 
by magnetometry. Finally, our findings are summarized in Sec. IV.

\section{JOSEPHSON COUPLING}

\section{A. Theoretical formalism}

It is widely accepted that the high critical temperature of $\mathrm{MgB}_{2}$ arises due to the coupling of the superconducting bands which effectively reinforce each other. However, the exact nature of the coupling is not fully understood, and possible scenarios are the exchange of electrons, Cooper pairs, interaction between the respective supercurrents, interaction through the internal magnetic field, etc. Microscopic $a b$ initio calculations have not been able to pinpoint the key interaction. In what follows, we will consider the Josephson coupling between the bands, resulting from the tunneling of the Cooper pairs from one band to another. This is incorporated in the Ginzburg-Landau (GL) energy functional ${ }^{8}$ through an interaction term dependent on the order parameter of both bands and proportional to $\Gamma$, the Josephson coupling strength,

$$
\begin{aligned}
\Delta F= & \int\left\{\sum _ { n = 1 } ^ { 2 } \left[\frac{1}{2 m_{n}}\left|\left(-i \hbar \nabla-\frac{2 e}{c} \vec{A}\right) \Psi_{n}\right|^{2}+\alpha_{n}\left|\Psi_{n}\right|^{2}\right.\right. \\
& \left.\left.\left.+\frac{1}{2} \beta_{n}\left|\Psi_{n}\right|^{4}\right]-\Gamma\left(\Psi_{1}^{*} \Psi_{2}+\Psi_{2}^{*} \Psi_{1}\right)\right]\right\} d V,
\end{aligned}
$$

where $\alpha_{n}=\alpha_{n 0}\left(1-T / T_{c n}\right)$ and $\beta_{n}$ are the GL coefficients, and $n$ is the band index. This results in a set of nine parameters describing a two-gap system: $\alpha_{10}, \alpha_{20}, \beta_{1}, \beta_{2}$, the Cooperpair mass $m_{1}$ and $m_{2}, \Gamma$, and the critical temperatures $T_{c 1}$ and $T_{c 2}$. While each band has its own intrinsic critical temperature, Josephson coupling causes both bands to survive up to a higher critical temperature, $T_{c}>\max \left(T_{c 1}, T_{c 2}\right)$. While the GL functional is derived for $T \lesssim T_{c}$, experience with mesoscopic single-gap superconductors indicates that the GL equations in practice are valid much deeper into the superconducting state. In Eq. (1) we then introduce temperature independent units, in order to rewrite it in a dimensionless form. We express the free energy of the system in units of $F_{10}=\alpha_{10}^{2} / \beta_{1}$, length in units of $\xi_{10}\left(\xi_{n 0}=\hbar / \sqrt{-2 m_{n} \alpha_{n 0}}\right)$, the vector potential in $A_{0}=\hbar c / 2 e \xi_{10}$, the order parameters in $\Psi_{n 0}=\Psi_{n 0}(T=0, \Gamma=0, H=0)=\sqrt{-\alpha_{n 0} / \beta_{n}}$ and the temperature in $T_{c 1}$. This reduces the set of necessary parameters to five: $\delta=\Psi_{10} / \Psi_{20}, \quad \alpha=\xi_{10}^{2} / \xi_{20}^{2}, \quad m=m_{1} / m_{2}, \quad T_{c r}$ $=T_{c 2} / T_{c 1}$, and $\gamma=\Gamma / \alpha_{10}$. In addition, we have two external tunable parameters: the temperature $T$ and the applied field $H$. The flux $\phi$ is defined as the externally applied flux. We first consider an extreme type-II case, and neglect the selfinduced magnetic field in the sample.

The minimization of the energy functional leads to the two-band GL equations. After the scaling described above, the equations for the order parameters read:

$$
\left\{\begin{array}{r}
(-i \nabla-\vec{A})^{2} \psi_{1}-\left(1-T-\left|\psi_{1}\right|^{2}\right) \psi_{1}-\frac{\gamma}{\delta} \psi_{2}=0, \\
\frac{1}{\alpha}(-i \nabla-\vec{A})^{2} \psi_{2}-\left(1-\frac{T}{T_{c r}}-\left|\psi_{2}\right|^{2}\right) \psi_{2}-\frac{\gamma \delta}{m \alpha} \psi_{1}=0 .
\end{array}\right.
$$

This system of nonlinear coupled differential equations we solve numerically on a square grid of typically 128 $\times 128$ points. The details of this procedure can be found in Ref. 20.

In the following analysis we neglect the screening of the magnetic field. This is justified for an extreme type-II material, or any sufficiently thin sample. An applied vector potential $A=\left(\frac{1}{2} H y,-\frac{1}{2} H x, 0\right)$ results in a magnetic response resulting from a total supercurrent,

$$
\vec{j}_{s}=\Re\left[\psi_{1}(i \nabla-\vec{A}) \psi_{1}^{*}\right]+\frac{m}{\delta^{2}} \mathfrak{R}\left[\psi_{2}(i \nabla-\vec{A}) \psi_{2}^{*}\right] .
$$

The free energy functional in dimensionless units reads

$$
\begin{aligned}
\frac{\Delta F}{\alpha_{10}^{2} / \beta_{1}}= & \int\left[\left|(-i \nabla-\vec{A}) \psi_{1}\right|^{2}-(1-T)\left|\psi_{1}\right|^{2}+\frac{1}{2}\left|\psi_{1}\right|^{4}\right. \\
& +\frac{m}{\delta^{2}}\left|(-i \nabla-\vec{A}) \psi_{2}\right|^{2}+\frac{m \alpha}{\delta^{2}}\left(-\left(1-T / T_{c r}\right)\left|\psi_{2}\right|^{2}\right. \\
& \left.\left.\left.+\frac{1}{2}\left|\psi_{2}\right|^{4}\right)-\frac{\gamma}{\delta}\left(\psi_{1}^{*} \psi_{2}+\psi_{2}^{*} \psi_{1}\right)\right)\right] d V .
\end{aligned}
$$

Let us here address several direct implications of Josephson coupling. It is clear from Eq. (1) that the sign of $\gamma$ determines the relative phase shift between the order parameters in the two condensates-either $\approx 0$ when $\gamma>0$ or $\approx \pi$ when $\gamma<0$-in order for the coupling term to provide a negative energy contribution. However, the sign of $\gamma$ has no influence on observables such as the Cooper-pair density and magnetic response of the sample. The general consequence of $\gamma$ coupling is an injection of Cooper pairs from one band into the other and vice versa, thus increasing the stability of the superconducting state. In other words, the average Cooper-pair density always increases with $\gamma$. In the absence of an applied field, the ratio $\chi=\psi_{1} / \psi_{2}$ can be found from

$$
\frac{\gamma \delta}{m \alpha} \chi^{4}+\left(1-\frac{T}{T_{c r}}\right) \chi^{3}-(1-T) \chi-\frac{\gamma}{\delta}=0,
$$

analytically derived from the GL equations. From this we find that in the limit $\gamma \rightarrow \infty$ a constant ratio $\psi_{1} / \psi_{2}(\mathrm{H}=0)$ $=\sqrt{\sqrt{m \alpha} / \delta}$ is reached, independent of temperature.

The next section is dedicated to an analysis of the size of the vortex core. Before we get into the physics of the problem, we here address some numerical issues following from mapping of the superconducting disk on a square numerical grid. For obvious reasons, the influence of the resolution of the numerical grid $N$, on the observed vortex size $R_{V}$, is significant. In Fig. 1(a) we show the $R_{V}$ vs $N$ (definition of $R_{V}$ is given in the next section). With increasing grid density, the numerical error decreases, and the vortex size converges toward the $R_{0}$ value with dependence $R_{V}=R_{0}+b / N$. By a 


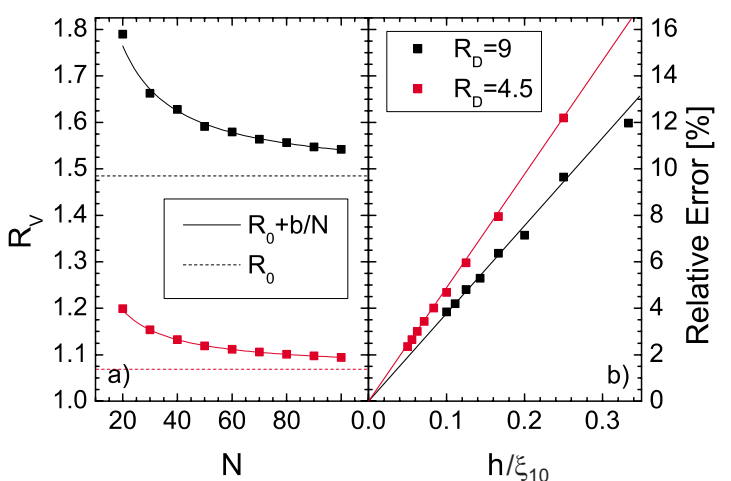

FIG. 1. (Color online) (a) The dependence of the observed vortex size $R_{V}$ on the number of grid points in the numerical mesh $N$. (b) The relative error in found vortex size vs grid spacing $h$, for two different sizes of the superconducting disk.

fitting procedure $R_{0}$ and $b$ can be determined and the corresponding curves are represented by the solid lines in the figure. The dashed lines are the asymptotes with value $R_{0}$. In Fig. 1(b) the relative error is plotted as a function of $h$, the grid spacing (proportional to sample size and inversely proportional to $N$ ). We conclude that due to numerics the vortex size is always slightly overestimated, with overshoot increasing for smaller samples (where influence of the boundaries is more pronounced). Nevertheless, with a resolution of 10 points per coherence length we get a relative error under $5 \%$. Although higher grid density obviously improves the results, we refrain from using a density above 10 points/ $\xi$, in order to optimize the speed of the calculation.

\section{B. Size of the vortex core}

The coherence length is the characteristic length scale over which the order parameter changes. It is therefore intuitive that the size of the vortex core is proportional to the coherence length in bulk superconductors. In single-gap materials, the coherence length is proportional to $1 / \sqrt{1-T / T_{c}}$ in the temperature range where the Ginzburg-Landau theory is valid. Here we show that in two-gap superconductors the coherence length is strongly affected by the coupling parameter $\gamma$, generally in an opposite manner from temperature. For comparison, we can use the coherence length obtained from the expression for the second critical field derived in Ref. 8, through the relation $H_{c 2}=\Phi_{0} / 2 \pi \xi^{2}$, with $\Phi_{0}$ being the flux quantum. Deviations are a priori expected, since already experiments of Refs. 6 and 7 found a discrepancy between the vortex size and the coherence length deduced from the second critical field.

To estimate the coherence length, we will numerically determine the size of the vortex core, a quantity which is not uniquely defined. In the following calculations, we examine the single-vortex state in $\mathrm{a} \mathrm{MB}_{2}$ superconducting disk exposed to a field providing three flux quanta through the sample. For the definition of the vortex size, two possibilities are considered in the literature: (i) the vortex size is determined by the distance from the center of the vortex to the contour where the Cooper-pair density (CPD) recovers to some percentage of its maximal value in the sample, denoted

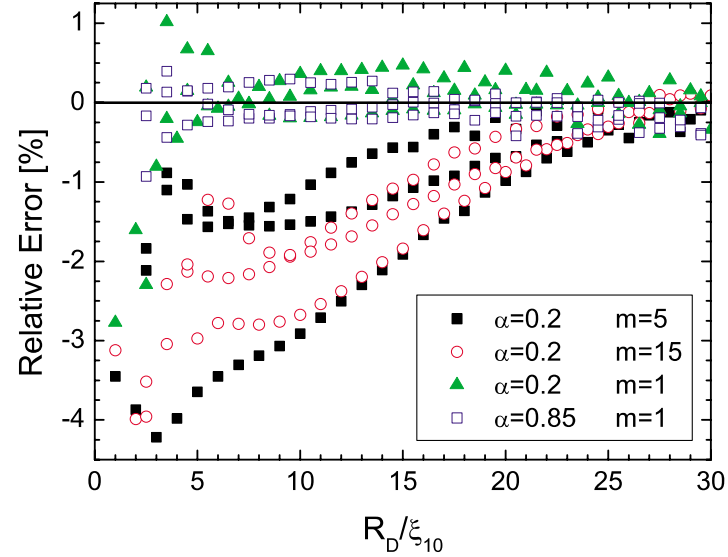

FIG. 2. (Color online) The relative error of the result of Eq. (6) compared to the observed vortex size, as a function of disk size, for indicated different values of parameters $m$ and $\alpha$, each with three different combinations of $\gamma$ and $T$ in order to cover as large as possible parameter space in the analysis.

as $R_{V, C P D}$; and (ii) the vortex size is the distance from the center of the vortex to the first contour where the supercurrent $j_{s}$ reaches its maximum, denoted as $R_{V, j}$. The problem of the first definition is the arbitrary threshold value for the criterion, but also the fact that we have two Cooper-pair densities, which makes the single vortex size ambiguous. As a threshold we take $80 \%$, since this allows for a more precise vortex size determination, and we will consider only the first condensate. On the other hand, the second definition involves coupled condensates and thus provides us with a unique vortex size. We therefore adopt the second definition to describe the vortex size in the rest of this work. Contrary to the bulk case, in our mesoscopic disks both definitions render a vortex size dependent on the radius of the disk $R_{D}$, as vortex currents in the center of the sample can interact with Meissner currents decaying from the edge inward. However, while $R_{V, C P D}$ in each band saturates for $R_{D} \rightarrow \infty$, this is not the case for $R_{V, j}$ : it develops a linear dependence on $R_{D}$. We extracted the exact dependence of both definitions of the vortex size $R_{V}$ on the disk size $R_{D}$, which led us to a universal formula (valid for both definitions, but with different coefficients),

$$
\left(\frac{1}{R_{V 0}}\right)^{2}+\left(\frac{c}{R_{D}}\right)^{2}=\left(\frac{1}{R_{V}-h R_{D}}\right)^{2},
$$

where $R_{V 0}$ is the vortex size independent of the sample size, $c$ is a length coefficient and $h$ is the slope of $R_{V}$ vs $R_{D}$ for large $R_{D}$. From fitting of our numerical data, collected at different $T, \gamma, \alpha$, and $m$, we obtained $\{c=1.90, h=0\}$ for $R_{V, C P D}$ and $\{c=3.40, h=0.006\}$ for $R_{V, j}$. These coefficients are valid for single gap superconductors as well as for twogap superconductors, even when the coherence lengths of the two condensates are very different (e.g., for small $\alpha$, see Fig. 2 ). The function gives an excellent estimate of the vortex size for $m \neq 1$, for disks larger than $5 \xi$. As shown in Fig. 2, deviation from the given function does occurs for specific choices of $m$ and $\alpha$, especially for small disks, but the relative error remains under $5 \%$. 


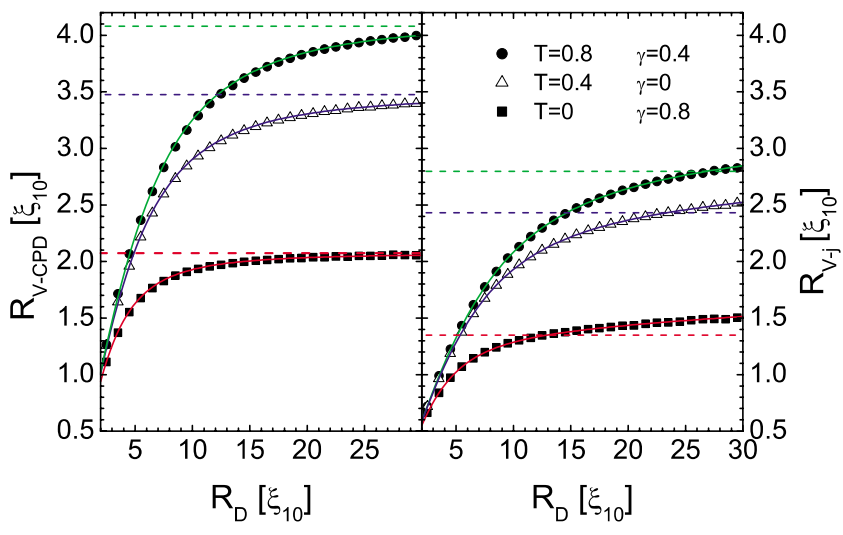

FIG. 3. (Color online) Vortex size, determined through the decay of the Cooper-pair density $R_{V-C P D}$ (a) and determined through the maximum of encircling currents $R_{V-j}$ (b), as a function of the radius of the superconducting disk $R_{D}$. The solid dots represent the numerical results, while solid lines show the fit using Eq. (6). The dashed lines indicate $R_{V 0}$, the fitting parameter corresponding to the sample-independent vortex size.

Both previously given definitions of $R_{V}$ are illustrated in Fig. 3. When disks are too small, the vortex size is not always fixed by the disk size only, e.g., when $T>T_{c r}$ and $\gamma$ $<0.1$, when the coherence lengths differ much and the interaction of the vortex with the Meissner currents becomes too different in the two bands. We found however that when $R_{D}>10 \xi_{10}$ these mesoscopic effects have only a minor influence and the correspondence between $R_{V}$ and $R_{D}$ becomes predictable again.

With the established dependence of the vortex size on the size of the sample, we can more precisely determine the actual influence of the coupling $\gamma$ on the vortex size. In particular we will look at the behavior of $R_{V 0, j}$ as a function of $T, T_{c r}$, and $\gamma$ (parameters $\alpha$ and $m$ remain fixed at realistic values for $\left.\mathrm{MgB}_{2}\right)$.

In Fig. 4 the size-independent vortex size $R_{V 0}$ is plotted versus $\gamma$, for different temperatures $T$ and $T_{c r}$. The dots represent the result of our simulations. The general behavior can be described by the following observed trends: (i) increasing $T$ induces an increase of the vortex size whereas increasing $\gamma$ has the opposite effect. (ii) Deviation from the latter mono-

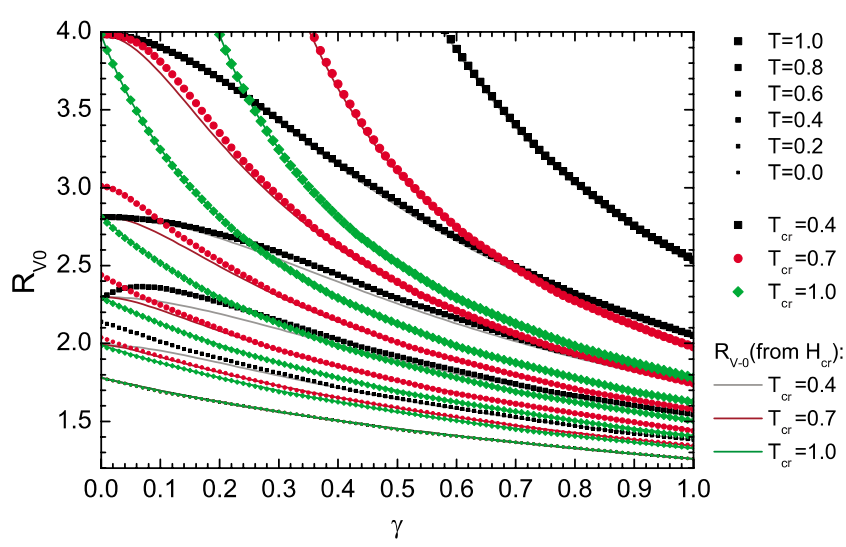

FIG. 4. (Color online) $R_{V 0}$ as a function of $\gamma$ and temperature, for $\gamma<1$ and for different values of $T_{c 2} . \alpha=m=1$. tonic behavior occurs when $T \approx T_{c r}$ and the coupling is weak, see, e.g., the curve at $T=T_{c r}=0.4$. The reason for the initial positive slope is that the second band is revived by the presence of coupling but retains its own character (i.e., a larger coherence length) since coupling is still weak. (iii) For $T$ $>T_{c r}$, curves with different $T_{c r}$ but identical $T$ merge at $\gamma$ $=0$ since then only the first condensate survives and fully determines the vortex size. In Fig. 4, the solid curves represent an estimate of the vortex size based on the general relation between the coherence length and the upper critical field in a single gap bulk superconductor $H_{c 2} \propto 1 / \xi^{2}$,

$$
\xi=\frac{\sqrt{2}}{\sqrt{g_{+}\left(\alpha, T, T_{c r}\right)+\sqrt{g_{-}\left(\alpha, T, T_{c r}\right)^{2}+4 \frac{\gamma^{2}}{m}}}},
$$

with $g_{ \pm}\left(\alpha, T, T_{c r}\right)=1-T \pm \alpha\left(1-\frac{T}{T_{c r}}\right)$, based on the analytical expression for the critical field of a bulk two-gap superconductor,

$$
\begin{aligned}
H_{c 2}(T) \propto 1-T+\alpha\left(1-\frac{T}{T_{c 2}}\right) \\
+\sqrt{\left[1-T-\alpha\left(1-\frac{T}{T_{c 2}}\right)\right]^{2}+4 \frac{\gamma^{2}}{m}},
\end{aligned}
$$

taken from Ref. 8. We find that the vortex size in our samples scales with the coherence length as $R_{V 0}=1.78 \xi$, which we use to plot the curves in Fig. 4. These theoretical curves coincide rather well with the data for $\gamma>0.25$. The reason for this is that, when coupling becomes sufficiently strong, both order parameters tend to have a similar spatial distribution and thus also exhibit a similar vortex size and coherence length. A good correspondence between the data and the fitted curves is also found for $T \gg T_{c r}$, i.e., when the second condensate exists solely due to the coupling to the first condensate, or in the case of weak coupling and the second condensate is almost depleted, so that it does not influence the vortex size. Two regions of discrepancy include $T \approx T_{c r}$ ( $R_{V 0}$ behaves nonmonotonic), and $T \ll T_{c r}$ and weak coupling. For the latter case, the formula still predicts $R_{V 0}(\gamma$ $=0)$ to be independent of $T_{c r}$, while this is clearly not the case. In this regime the vortex size is found to behave more like that of a single gap superconductor, but with a different critical temperature. By fitting we determined a function that describes the behavior of the vortex size accurately in this regime as

$$
R_{V 0}=\frac{1.78}{\sqrt{1-T / \sqrt{T_{c r}}+\gamma}} .
$$

This equation is generally applicable, and effectively shows our initial premise that $\gamma$ has an opposite influence to $T$.

We notice however that Eq. (7) also contains the dependence on $\alpha$ and $m$. However, this formula can not adequately describe the vortex size for $m, \alpha$ much different from 1 , since the properties of the two condensates can no longer be described by a single coherence length. In general we can state 


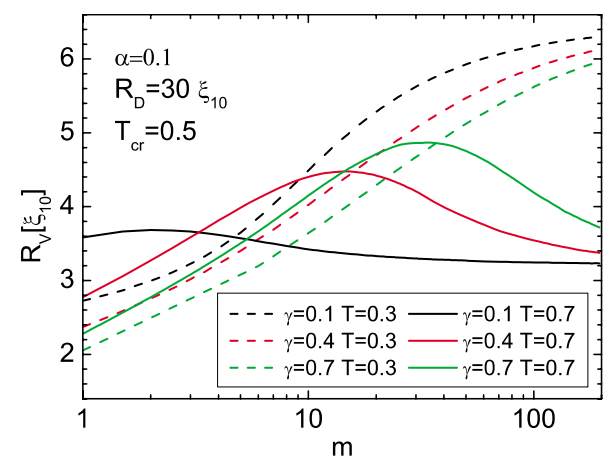

FIG. 5. (Color online) The apparent vortex size in a disk with radius $R=30 \xi$ as a function of $m$, for different $\gamma$ and $T$. The ratio of coherence lengths in two condensates is fixed at $\xi_{20} / \xi_{10}=3.162$, i.e., $\alpha=0.1$.

that the relation between the coherence length and the critical field does not hold anymore when the individual coherence lengths differ too strongly. In Fig. 5 we plot the numerically obtained $R_{V}$ as a function of $m$, for a small parameter $\alpha$ (with thus an acute difference between the coherence lengths in the two condensates). The analytic estimate of Eq. (7) is monotonously increasing with $m$ in this case, and is obviously not useful for comparison with nonmonotonically evolving curves in Fig. 5.

Let us first analyze the limiting case of extremely large $m$. Following from Eq. (2), the second condensate decouples from the first one in this limit. At the same time, as seen in Eq. (3), the influence of the second condensate on the total current in the system increases. Therefore, the size of the vortex $R_{V, j}$ will be fully determined by the second condensate [and its nominal coherence length $\xi_{2}(T)$ ], provided that the temperature is below $T_{c r}$. Otherwise, the vortex size is determined solely by the first condensate [and $\xi_{1}(T)$ ], since the coupling between the condensates is entirely suppressed and the second condensate fully depletes.

This helps us to understand the behavior of the vortex size as a function of $m$, shown in Fig. 5. At $T<T_{c r}$, the initial increase of $m$ decreases the coupling of the second condensate to the first ( $m$ has an opposite effect from $\gamma$, see Eq. (2). This causes an increase of the apparent vortex size, due to much larger coherence length of the second condensate ( $\alpha$ $=0.1$ ), and at the same time the increase of $m$ makes the supercurrent of the second band stronger and thus more deterministic for the magnetically detectable size of the vortex [see Eq. (3)]. Above $T_{c r}$ these two effects become competing, since the first will deplete the second condensate and therefore reduce its influence while the second enhances the influence of the second condensate. These competing effects result in the nonmonotonic behavior of the vortex size vs $m$ in Fig. 5. At low $m$, the influence of the large coherence length in the second condensate dominates, whereas at large $m$ the coupling disappears and the second condensate depletes. At very large $m$, all curves for $T>T_{c r}$ saturate to the same value, namely the size of the vortex core in the first condensate, in the absence of a second one.

In Fig. 6 we demonstrate some peculiarities of the dependence of the vortex size on the parameter $\alpha$. $\alpha$ was swept down from 1 to 0.005 in a disk with parameters $m=\delta=1$,

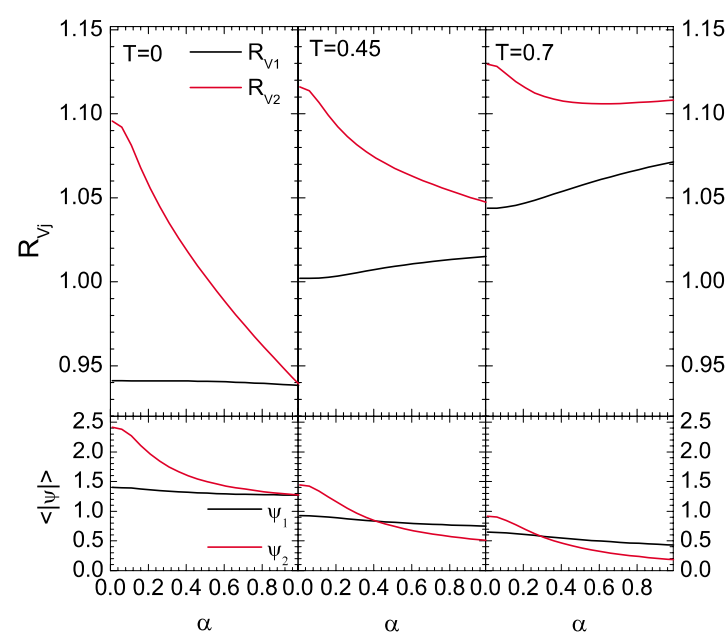

FIG. 6. (Color online) Top: $R_{V}$ in each condensate as a function of $\alpha$, for different $\gamma, T$. Bottom: ratio of condensation energies of the two condensates (logarithmic scale) vs $\alpha$. When equal to 1 , condensates influence each other with equal strength since $m=1$.

$T_{c r}=0.5$, and $R_{D}=4 \xi_{10}$. In the absence of coupling, this sweep increases $\xi_{20}$ while $\xi_{10}$ is kept constant, as the length unit of the GL equations. In the presence of coupling, both the resulting $\xi_{2}$ and the resulting $\xi_{1}$ will be influenced (i.e., the Cooper-pair correlation length in each of the condensates, different from the nominal coherence lengths in each condensate separately). Intuitively, one expects that coupling causes vortex cores in the two condensates to have similar behavior, and tend toward similar sizes; instead, for decreasing $\alpha$ at temperatures $T=0.45$ and $T=0.7$ an increase of $R_{V 2}$ is observed while $R_{V 1}$ decreases. For stronger coupling this effect becomes even more prominent, compared to the vortex size at $\alpha=1$. At lower temperatures (shown for $T=0$ in Fig. 6 ), the behavior of $R_{V, j}(\alpha)$ for $T=0$ is in better concordance with the intuition: $R_{V 1}$ increases as $R_{V 2}$ increases, with the effect growing with coupling. However this effect is weak.

To better understand the behavior of the vortex sizes we will invoke the full free energy expression of Eq. (4), which can be written as $F=F_{1}+F_{2}+F_{12}$, where $F_{i}$ depends only on $\psi_{i}$ and $F_{12}$ is the Josephson coupling term. The key point here is that a decrease of $\alpha$ will lower $F_{2}$ directly, therefore giving more weight to the other terms $F_{1}$ and $F_{12}$, i.e., changing $\alpha$ reorders the hierarchy of the terms. A stronger $F_{12}$ stimulates an increase of $\psi_{1}$ and $\psi_{2}$. Since $F_{2}$, which regulates the size of $\psi_{2}$, becomes less important, $\psi_{2}$ will increase much faster than $\psi_{1}$. A similar behavior of the $\psi$ and $R_{V}$ curves hints to the link between the two variables. For an infinite superconductor, sweeping $\alpha$ to zero would cause both $\psi_{1}$ and $\psi_{2}$ to diverge. However for a finite (mesoscopic) superconductor, the vortex size will eventually exceed the disk size, thereby effectively suppressing the order parameter and preventing the divergence.

The decrease of $R_{V 1}$ with decreasing $\alpha$ can be ascribed to the increase of $\psi_{1}$. However, in the left panel of Fig. 6 where temperature is zero, we notice the subtle increase of $R_{V 1}$ with decreasing $\alpha$. Here $\psi_{2}$ is still relatively large (because of the low temperature) which causes $\psi_{1}$ to feel a strong influence from $\psi_{2}$ due to coupling. As a result, for low $T$, vortex sizes 


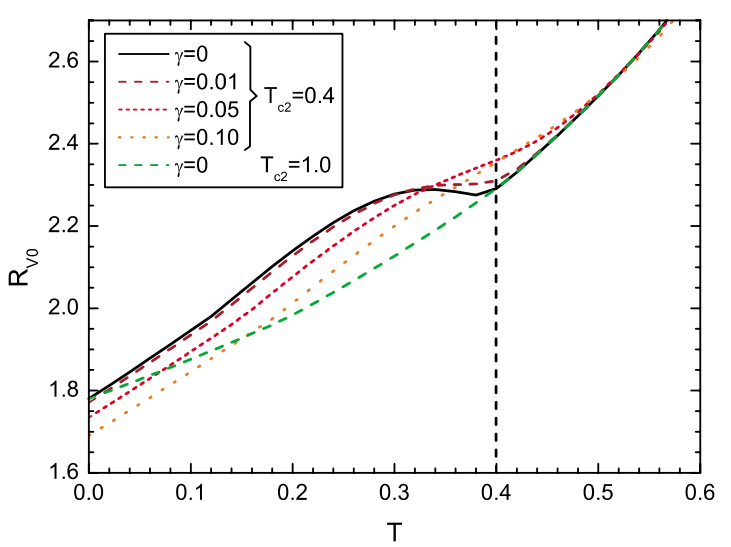

FIG. 7. (Color online) The size-independent vortex size $R_{V 0}$ as a function of temperature (for fixed $T_{c 1}$ and $T_{c 2}$ ), for several values of $\gamma . R_{D}=10 \xi_{0}, m=1$, and $\alpha=1$.

will tend to be similar in the two condensates, as we intuitively predicted. In the central and the right panel of Fig. 6, i.e., for higher temperatures, the influence of $\psi_{2}$ is reduced, and the increased order parameter $\psi_{1}$ prevails in determining the vortex size-thus $R_{V 1}$ decreases. In the right panel $T$ $>T_{2}$, and the second condensate would be depleted in the absence of coupling. This creates yet another regime of the $R_{V}(\alpha)$ dependence: initially $R_{V 2}$ now decreases with decreasing $\alpha$ from 1 . The second condensate is completely dependent on the first one, and therefore obeys its shape. When $\alpha$ further decreases, $\psi_{2}$ is less modified and an increase of $R_{V 2}$, is recovered.

Finally we point out one more interesting artifact. In Fig. 7 we show the calculated vortex size as a function of temperature, for very weak coupling, i.e., small $\gamma$, and $m=\alpha$ $=1$. The observed kink corresponds to the critical temperature of the second band, and smears out when $\gamma$ is increased. The $\left\{\gamma=0, T_{c r}=0.4\right\}$ curve starts at the same value as the $\left\{\gamma=0, T_{c r}=1\right\}$ curve, since at $T=0$ there is no dependence of vortex size on $T_{c r}$. This behavior is observable by magnetic force, scanning Hall probe, or scanning tunneling microscopy, and we expect its experimental verification.

For the $\left\{\gamma=0, T_{c r}=1\right\}$ curve, the superconducting state and supercurrents in both condensates are identical. Therefore the vortex size defined on separate condensates as well as on the combined system will be equal. For this reason the curve coincides with the $\left\{\gamma=0, T_{c 2}=0.4\right\}$ case for $T>T_{c 2}$ $=0.4$, since then the second band is depleted and only the first band superconducts.

\section{C. $H$-T phase diagrams}

In increasing magnetic field, more vortices penetrate the superconducting system. It is known that the symmetry of the vortex states is strongly affected by the symmetry of the mesoscopic sample, as detailed in Ref. 21. With increasing temperature, the symmetry of the sample is even stronger imposed on the vortex matter, and it is therefore no surprise that in mesoscopic disks most vortex state configurations collapse into a giant-vortex ${ }^{22}$ at high temperature. We can construct an $H$ - $T$ diagram for mesoscopic samples, indicat-

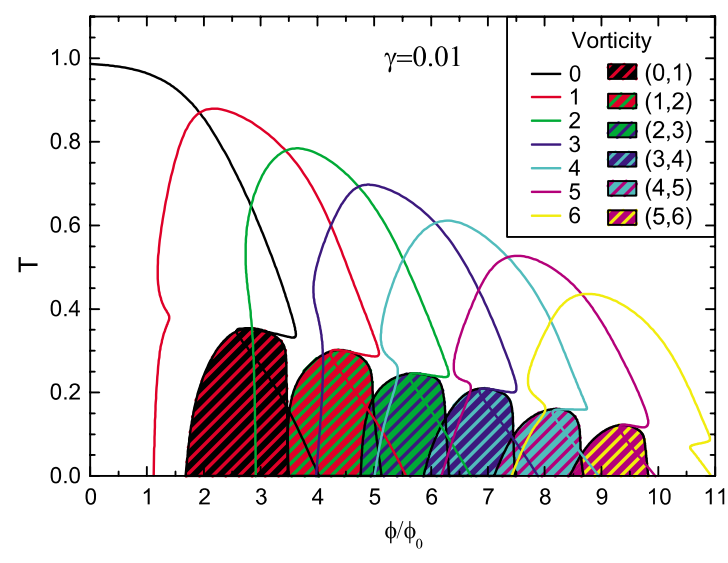

FIG. 8. (Color online) The magnetic flux-temperature stability regions for different vortex states (vorticity $L$ ) in a two-band disk of size $R_{D} / \xi_{10}=4$, and with $T_{c 2}=0.44, \delta=1.33, \alpha=0.844$, and $\gamma=0.01$. In color-coded areas, the vortex state is fractional and cannot be represented by a single $L$, but rather as $\left(L_{1}, L_{2}\right)$ state, where vorticities in two condensates are given, respectively.

ing the area of stability of states with different vorticity. Two-gap systems make there no exception, but do comprise several particularities. In Fig. 8 we displayed the full stability regions of all possible vortex states with vorticity $L<7$ in a superconducting disk with parameters $\left\{T_{c 2}=0.44, \delta\right.$ $=1.33, \alpha=0.844, m=1\},{ }^{19}$ which is very similar to $\mathrm{MgB}_{2}$ except for the coupling parameter, where we took significantly smaller $\gamma=0.01$. This choice provides more complexity to the vortex states, as it allows for different vorticities and vortex arrangements in the two bands.

Indeed, one difference from the single-gap superconducting disks is directly visible in Fig. 8, where the stability regions of composite vortex states are mushroom shaped. In other words, with increasing temperature, one can exit the stability range of a particular $L$ state, but then find it again at higher temperatures. This shape has the following origin: At high temperatures $\left(T_{c 2} \ll T<T_{c 1}\right)$ the second gap would be completely depleted if it was not for the coupling. In other words, the second band depends completely on the first band, and therefore has the same behavior and features like the $H(T)$ boundary. However, for low temperatures $\left(T \leqq T_{c 2}\right)$ the second band is still active and retains its own character, and therefore the stability region boundary follows quite closely the single-gap stability region.

In the mushroom-shaped areas, the vorticity in the two bands is the same. However, in the shaded areas we found vortex states where the vorticity differs from one band to the other. As a consequence, the overall, apparent vorticity of the sample is no longer integer! These are the so-called fractional vortex states.

As clearly shown in Fig. 9(a), the full $H$ - $T$ stability region of an integer flux vortex state is related to the union of the $H-T$ stability regions for the given state in the two corresponding single-gap condensates. On the other hand, the fractional states are found at the intersection of two corresponding single-gap stability regions [see Fig. 9(b), for the $\left(L_{1}, L_{2}\right)=(4,5)$ state $]$. 


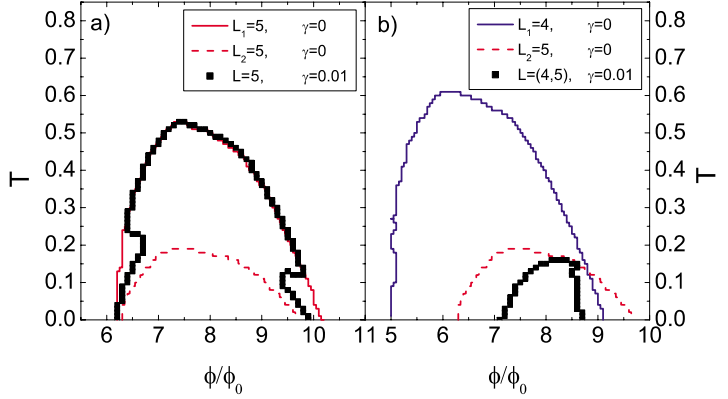

FIG. 9. (Color online) Illustration on how the stability fluxtemperature regions of integer (a) and fractional states (b) arise from the single-gap picture.

With increasing coupling parameter $\gamma$, the vortex states in the two condensates are linked together, and moreover reinforce each other. In Fig. 10 we show the stability region of the integer flux $L=4$ state, for three values of $\gamma$, where the $H-T$ stability region grows with $\gamma$. We conclude that increasing $\gamma$ stabilizes the integer flux states, but for the same reason destabilizes the fractional states. We discuss the latter further in the following section.

\section{Fractional vortex states}

The existence of fractional states, i.e., states with different vorticity in the bands, depends strongly on the coupling. They survive only at weak Josephson coupling between the bands, while only integer flux states are possible at large $\gamma$ values. This is illustrated in Fig. 11, where we show that the region of stability of fractional states shrinks with increasing coupling, but also that lower vorticity fractional states are more resilient to $\gamma$. Another interesting aspect of fractional states is their strong affinity to asymmetry. In both condensates vortices attempt to form a symmetric shell, but due to coupling and different respective number of vortices, the final state becomes asymmetric in most cases. For that reason, the asymmetry is more apparent at larger coupling $\gamma$. We show several examples through the log plots of the Cooperpair density of the chosen states in Fig. 11. Note that the fractional state not necessarily contains vortices in both condensates; for example, inset 1 in Fig. 11 is the $(0,1)$ state. Due to coupling, the total energy is minimized when regions

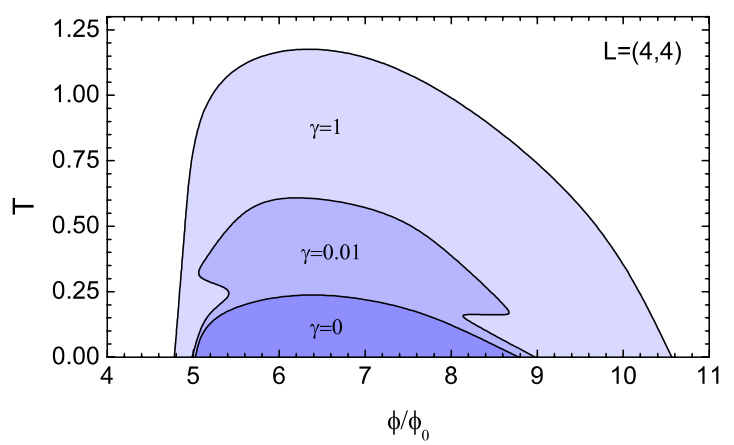

FIG. 10. (Color online) The stability region of the $L=4$ integer flux vortex state, for three different strengths of coupling $\gamma$.
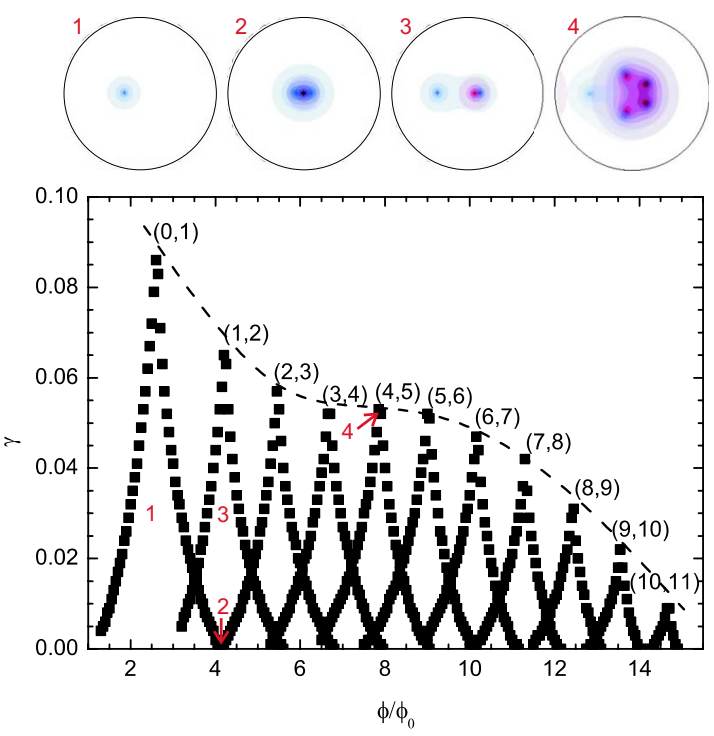

FIG. 11. (Color online) The stability regions in $\phi-\gamma$ parameter space of fractional states with different vorticities in the two bands. Parameters of the sample are $R_{D} / \xi_{10}=4, \alpha=\delta=m=1$ and $T=0$. In insets at the top of the figure, we superimposed the logarithmic plots of the Cooper-pair density in the two gaps on each other (red/blue shades for condensates $1 / 2$, respectively) for states indicated in the phase diagram by the red numbers.

with depleted order parameter in two condensates are on top of each other. As a result, the vortex of the second band is attracted to the boundary of the sample, where the circulating Meissner currents strongly suppress the order parameter in the first band. Inset 2 is the $(1,2)$ state for $\gamma=0$, i.e., the condensates are decoupled. This fractional state is therefore twofold symmetric, but when we increase $\gamma$ we enhance the asymmetric $(1,2)$ state, as shown in inset 3 . One vortex of the second band is attracted to the vortex of the first band, and the other is attracted to the edge of the sample. Finally we show in inset 4 the $(4,5)$ state, at the verge of its stability region, showing maximally pronounced asymmetry. Four vortices in both bands sit on top of each other, and the remaining, fifth vortex of the second band, breaks the symmetry and is gradually pulled out of the sample. The found states look similar to what was found earlier for Coulomb bound classical particles, ${ }^{23}$ although underlying physics is very different.

A two-gap mesoscopic system is a prime example of a vortex system with competing interactions. Besides the vortex-vortex interactions in each band, one must take into account the coupling between order parameters across the bands, and the mesoscopic effect of the compression of vortices to the interior by the circulating Meissner current that is maximal at the edge. For example, consider the $(0,1)$ state, where an outward force originates from the coupling between the vortex in the second condensate and the suppression of superconductivity at the edge of the first one. However, this action competes with the inward force exerted by the Meissner current. This purely mesoscopic effect leads to a tunable position of the vortex in this fractional state: while the Meissner current is roughly the same at a given magnetic field, the changed coupling between the condensates brings 


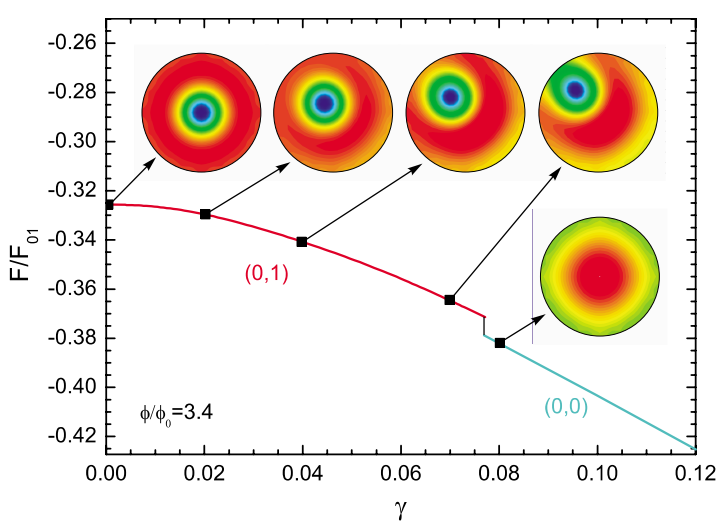

FIG. 12. (Color online) Calculated free energy of the fractional $(0,1)$ state, as a function of Josephson coupling between the bands. Insets show contour plots of the Cooper-pair density in the second gap, illustrating how the asymmetry gradually increases with $\gamma$ for taken $\mathrm{MgB}_{2}$ parameters and size of the disk $R_{D}=5 \xi_{10}$. The $\gamma$ sweep was done along the vertical dashed line in Fig. 13.

the vortex further to the boundary. This is shown in Fig. 12, as a transition from a fractional $(0,1)$ vortex state to an integer $L=0$ vortex state with increasing coupling.

As fascinating as they are, the fractional states are difficult to find in the ground state. For example, when the coherence lengths of the two bands are the same, then the energy landscape in both bands-considered as separate singlegap superconductors-are proportional, i.e., $F_{1}=\alpha \delta^{2} / m F_{2}$. All possible vortex states thus have their ground state in the same phase space region. The total energy of the system, $F$ $=F_{1}+F_{2}$, will therefore be proportional to the single-gap energy with as a direct consequence that fractional states always will have higher (or equal) energy compared to the integer states. To realize fractional states as the ground state, one therefore needs to make the discrepancy between the coherence lengths as large as possible. This can be done by taking $\alpha$ significantly different from one, or by taking temperature close to $T_{c 2}$, when $T_{c 1}>T_{c 2}$. In Fig. 13 we show the stability and ground state regions of the noncomposite states in a disk of size $R=5 \xi_{10}$ at temperature $T=0.4$ (and $\alpha=0.5$ ).

These asymmetric states can be observed in mesoscopic two-band samples. As main candidates for such an experiment, we select the imaging of only the $\pi$-band, as was done recently in Ref. 6. Alternatively, scanning Hall magnetometry or magnetic force microscopy can both reveal the asymmetric magnetic response of the sample in the case of a fractional state. We will revisit this point in the section devoted to magnetic coupling.

\section{E. Superconducting-normal phase boundary}

As mentioned in preceding sections, in Refs. 8 and 13 an expression was derived for the second critical field of a bulk two gap superconductor, given by Eq. (8). That expression contains the dependence of the critical field not just on $\gamma$, but on $m$ and $\alpha$ as well. We have shown that dependence does not describe the vortex properties in the two-band samples, but at this point we check its applicability for the estimation of the upper critical field of mesoscopic two-band disks at a

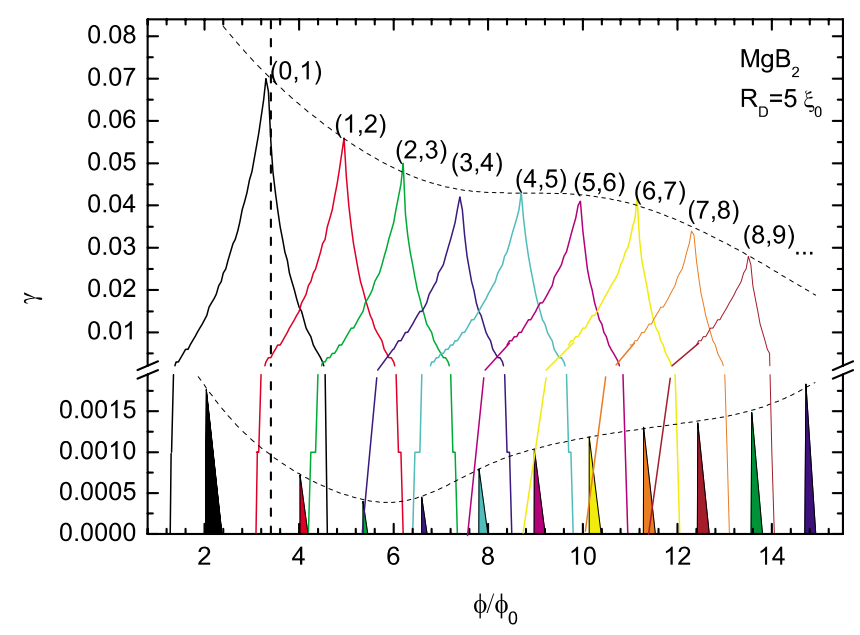

FIG. 13. (Color online) The stability regions and the ground state (colored) regions in $\phi-\gamma$-space of the fractional states. Taken parameters are $R_{D} / \xi_{10}=5, \alpha=0.844, \delta=1.33, m=1, T_{c 2}=0.44$, and $T=0.1$, corresponding to $\mathrm{MgB}_{2}$.

given temperature. Our results for the dependence of the upper critical field on the mass ratio in two bands are shown in Fig. 14(a), and demonstrate perfect agreement with Eq. (8), provided that the found critical field is scaled by its value at zero temperature and in absence of coupling.

Equation (8) is further applicable for the estimation of the $H-T$ superconducting-to-normal phase boundary. Namely, equating that expression to zero gives the expression for the critical temperature of the two-band sample,

$$
T_{c}=\frac{1}{2}\left(1+T_{c r}+\sqrt{\left(1-T_{c r}\right)^{2}+4 \frac{\gamma^{2}}{m \alpha} T_{c r}}\right) .
$$

This means that the critical temperature of a two gap superconductor is always equal or higher than the sum of the critical temperatures of the two bands, in the case as if there was no coupling. This observation is in contradiction with findings of Ref. 24, where it is claimed that also a lower $T_{c}$ is

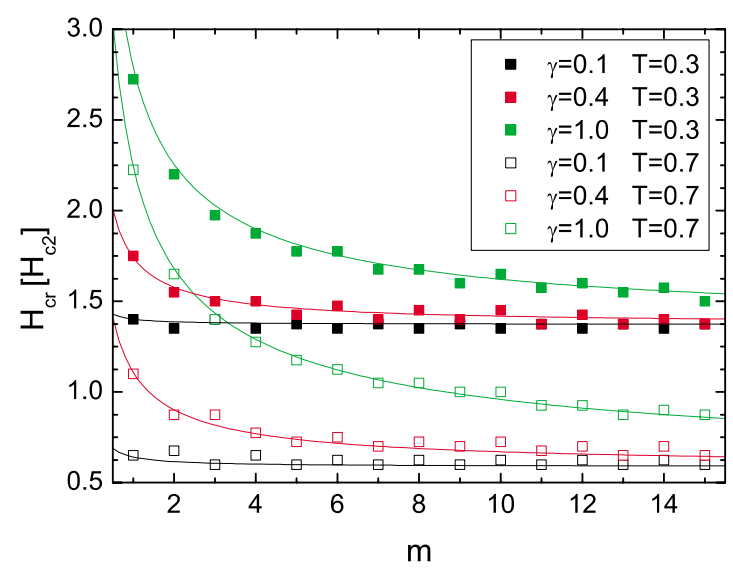

FIG. 14. (Color online) (a) The critical field of the mesoscopic disk $H_{c r}$ as a function of the mass ratio in two condensates. Parameters used are $R_{D}=4 \xi_{10}, T_{c 2}=0.5, \alpha=0.1$. (b) Idem but now as a function of $\alpha$. 


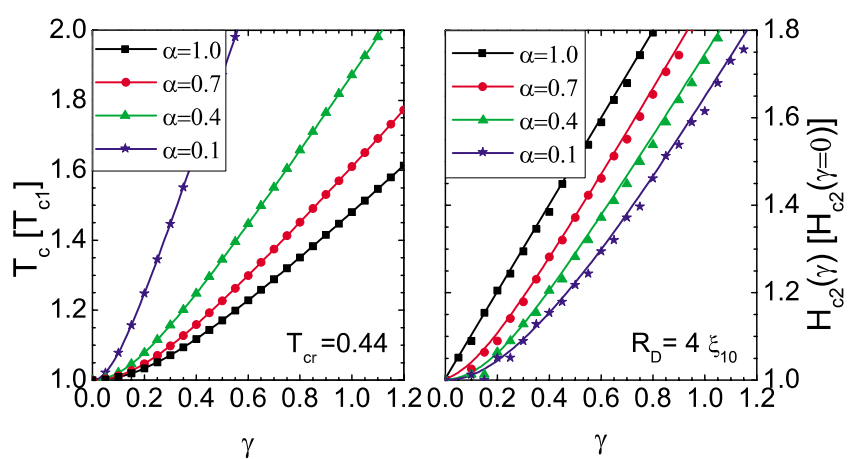

FIG. 15. (Color online) (a) The critical temperature $T_{c r}$ of a two-band mesoscopic disk as a function of Josephson coupling $\gamma$, in absence of magnetic field. (b) The upper critical field $H_{c r}$ vs $\gamma$ for $T=0$. Dots represent the numerical data, and the solid line is the result of Eqs. (8)-(10).

possible, depending on the parameters. The authors obtained these results from a microscopic derivation.

Above expressions were originally derived for bulk samples. It is already known that the upper critical field in mesoscopic superconductors is higher than in bulk, ${ }^{25}$ and it is therefore intuitively clear that Eq. (8) would not work for the case of two-gap mesoscopic disks. In Fig. 15 we show the numerically obtained critical temperature $T_{c r}$ and upper critical field $H_{c r}$ (corresponding to bulk $H_{c 2}$ ) versus $\gamma$ in disks of size $R=4 \xi_{10}$. We found that both the dependence of critical temperature and field on $\gamma$ obey the dependencies given in Eqs. (8)-(10), provided that the critical field is scaled to its value in the absence of coupling and at zero temperature. In Fig. 16 we show the calculated $T_{c}(H)$ boundary for different coupling strengths. Equation (8) can also be inverted to describe the dependence of $T_{c}$ on the applied field. Although derived for bulk, we find that latter equation nicely fits the $H(T)$ curves in Fig. 16 for a mesoscopic disk, after the aforementioned scaling of the magnetic field.

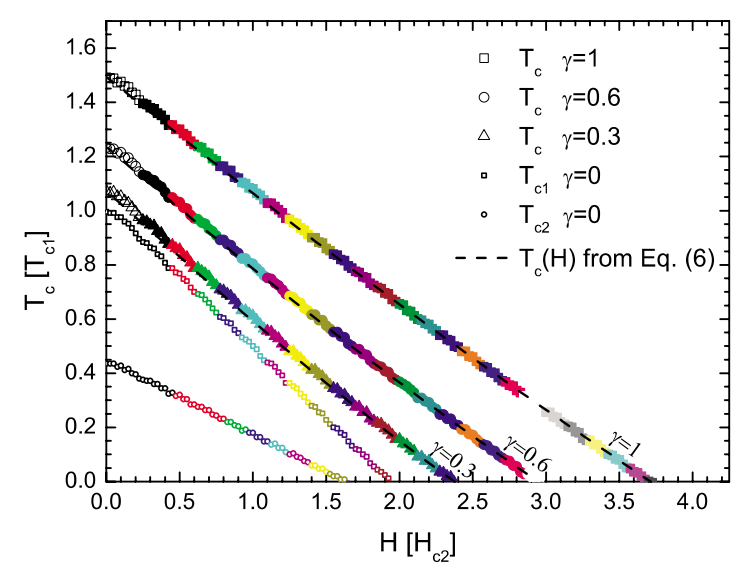

FIG. 16. (Color online) The superconducting-normal phase $H(T)$ boundary for the sample with parameters $R_{D}=4 \xi_{0}, \delta=1.33$, $\alpha=0.844, T_{c 2}=0.44$, and $m=1$, for different values of the Josephson coupling strength. Different colors of the dots mean different vorticities. Dashed lines are obtained from Eq. (8) with a prefactor described in the text.

\section{MAGNETIC COUPLING}

In the previous section, we assumed the existence of a Josephson coupling between two superconducting bands, but we neglected the screening of the magnetic field. In applied magnetic field, the magnetic response of a two-band superconductor follows from the induced supercurrent:

$$
\begin{aligned}
-\kappa_{1}^{2} \Delta \vec{A} & =\vec{j}_{s} \\
& =\Re\left[\psi_{1}(i \nabla-\vec{A}) \psi_{1}^{*}\right]+\frac{m}{\delta^{2}} \mathfrak{R}\left[\psi_{2}(i \nabla-\vec{A}) \psi_{2}^{*}\right] .
\end{aligned}
$$

Conventionally, the demagnetization and screening effects in mesoscopic superconductors are expressed through the Ginzburg-Landau parameter $\kappa$, being equal to the ratio of penetration depth $\lambda$ and coherence length $\xi$. For that reason, we reformulate the equations of Sec. II A to introduce $\kappa_{2}$, the GL parameter of the second condensate instead of the parameter $\delta$, the ratio of the order parameters in two bands. We start from the definitions of $\xi$ and $\lambda$,

$$
\lambda_{n 0}^{2}=\frac{m_{n} c^{2} \beta_{n}}{16 \pi \alpha_{n 0} e^{2}} \quad \xi_{n 0}^{2}=\frac{\hbar^{2}}{2 m_{n} \alpha_{n 0}},
$$

to derive

$$
\frac{\kappa_{1}^{2}}{\kappa_{2}^{2}}=\frac{m}{\delta^{2} \alpha},
$$

which we then substitute in the GL equations to obtain

$$
\begin{gathered}
(-i \nabla-\vec{A})^{2} \psi_{1}-\left(1-T-\left|\psi_{1}\right|^{2}\right) \psi_{1}=\frac{\gamma \alpha}{m} \frac{\kappa_{1}}{\kappa_{2}} \psi_{2}, \\
(-i \nabla-\vec{A})^{2} \psi_{2}-\alpha\left(1-\frac{T}{T_{c 2}}-\left|\psi_{2}\right|^{2}\right) \psi_{2}=\frac{\gamma}{\sqrt{m \alpha}} \frac{\kappa_{2}}{\kappa_{1}} \psi_{1},
\end{gathered}
$$

$$
-\Delta \vec{A}=\vec{j}_{s}=\frac{1}{\kappa_{1}^{2}} \mathfrak{R}\left[\psi_{1}(i \nabla-\vec{A}) \psi_{1}^{*}\right]+\frac{\alpha}{\kappa_{2}^{2}} \mathfrak{R}\left[\psi_{2}(i \nabla-\vec{A}) \psi_{2}^{*}\right]
$$

This form of two-band Ginzburg-Landau equations is particularly convenient for comparison with the conventional types of superconductivity. In the single-gap bulk samples, the value of $\kappa$ above or below $1 / \sqrt{2}$ determines the superconductor being of second or first type, respectively. For a two-band sample, this distinction is much more difficult to establish, since Eqs. (13)-(15) show the direct influence of not only $\kappa_{1}$ and $\kappa_{2}$, but also the Josephson coupling and the squared ratio of coherence lengths in two condensates $\alpha$.

Equation (15) also shows that two bands are directly coupled through the screening currents, and this type of coupling we refer to as magnetic coupling. In Fig. 17 we show the calculated magnetization of the disk with radius $R$ $=10 \xi_{10}$ as a function of the applied field (in absence of Josephson coupling), to illustrate how the magnetic field couples the two bands. We observe: (i) the magnetization of 


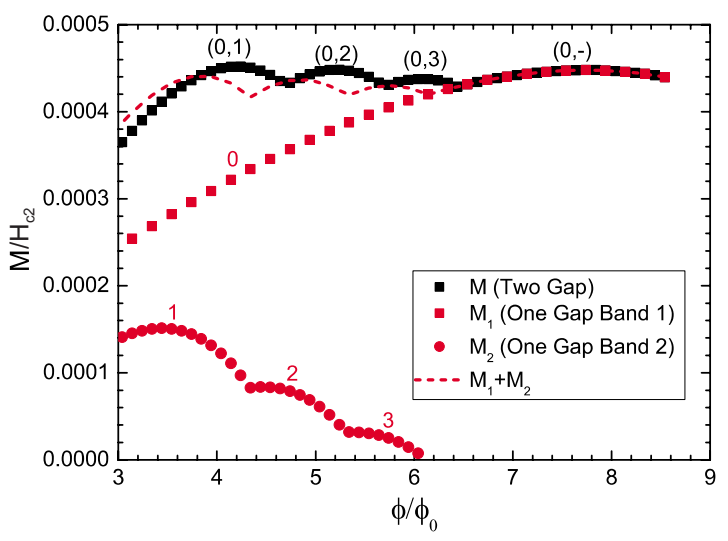

FIG. 17. (Color online) Magnetization versus applied magnetic field $[M(H)]$ loops for a two-gap disk with radius $R_{D}=10 \xi_{10}$, and two condensates as single-gap samples, in the absence of Josephson coupling. Magnetic coupling is included in the calculations, with parameters $\kappa_{1}=3.68, \kappa_{2}=0.66$, and $\alpha=0.06$.

the coupled system is somewhat higher than the sum of the two uncoupled systems; (ii) the found cusps are wider, since the Meissner currents in one band screens the field in the other band and flux entry is therefore more difficult. The net field in the interior of the sample is therefore lower, and the critical field is increased. This is in accordance with existing experimental findings on $\mathrm{MgB}_{2}$.

However, the information available in literature is also often confusing. For example, we identified two sets of parameters, both believed to be correct for $\mathrm{MgB}_{2}$. From Refs. 14 and 16 we extracted $\kappa_{1}=3.68, \kappa_{2}=0.66, \alpha=0.068$ which should be valid for a clean sample (single crystal). In these works the strength of the Josephson coupling, $\gamma$, is not estimated. In the dirty limit, the compound is definitely a type-II material. Substituting former values into Eq. (12) we obtain $\delta^{2} / m \approx 0.59$. On the other hand, from Refs. 8 and 19 we obtain $T_{c 2}=0.56 T_{c 1}$, and $\delta=1.33$. For usual $\mathrm{Mg}^{11} \mathrm{~B}_{2}$ we have $\gamma=0.4, m=1$, and $\alpha=0.844$. For the $\mathrm{Mg}^{10} \mathrm{~B}_{2}$ we found $\gamma$ $=0.28$. For irradiated $\mathrm{MgB}_{2}$ samples a mass ratio $m \approx 14$ has been observed together with $\alpha=0.059 .{ }^{19}$ Therefore, in the remainder of the paper, we will not restrict ourselves just to the particular values of the parameters, but rather focus on new physics between the two types of superconductivity and its manifestations.

\section{A. Magnetic vs Josephson coupling}

As mentioned above, one of the most fascinating properties of a two-band system is the possible appearance of fractional states. We argued that those could be observed in experiment through their magnetic response. Using the preceding theoretical formalism, we can now calculate the magnetic field in and around the sample, in response to the applied magnetic field. In Fig. 18 we show the magnetic field profile emanating from the mesoscopic superconducting disk in the fractional $(0,1)$ and $(1,2)$ vortex state. The asymmetry induced by the Josephson coupling is clearly visible and can be directly imaged in magnetic force microscopy or scanning Hall probe magnetometry experiments. Additionally, the integrated magnetic field from such measurements will reveal
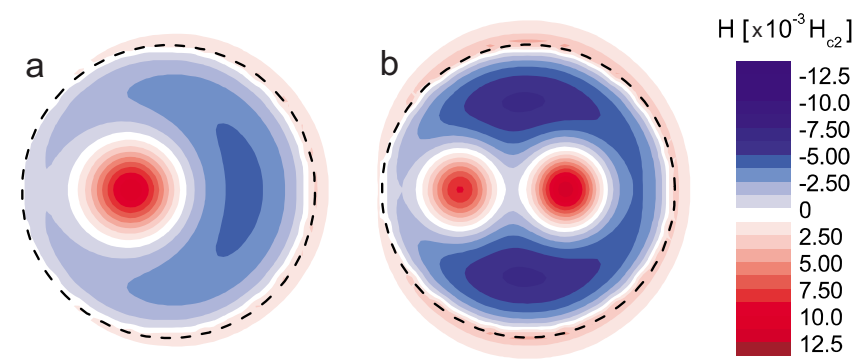

FIG. 18. (Color online) The magnetic response of the fractional $(0,1)$ and $(1,2)$ vortex states in a mesoscopic two gap superconducting disk with parameters $R_{D}=10 \xi_{0}, \kappa_{1}=10, \kappa_{2}=2, \alpha=0.3, \gamma=0.01$, and $T=0$. The applied field in (a) is $0.04 H_{c 2}\left(\phi / \phi_{0}=2\right)$ and in (b) $0.08 H_{c 2}\left(\phi / \phi_{0}=4\right)$. The dashed line shows the sample boundary.

the fractional flux carried by these states of noninteger total vorticity.

In Fig. 19 we show the effect of both Josephson and magnetic coupling on the free energy and the vortex states. Figure 19(a) shows the energy landscape when superconducting condensates are decoupled. As could be expected, all $(L, L$ $+1)$ fractional states are stabilized in the ground state, at intermediate fields between integer flux $L$ and $L+1$ states. $(L, L+2)$ states also exist, but have significantly higher energy. The same holds for $(L+1, L)$ fractional states, having far higher energy than the corresponding $(L, L+1)$ state. This can be entirely inverted for a different choice of parameters, particularly $\alpha$, which determines the relative coherence lengths and consequently the ratio of the vortex energy in the two bands. In the present calculation, we therefore omit the curves corresponding to $(L+1, L)$ states.

In Fig. 19(b) the Josephson coupling is added. This directly results in stabilization of the integer flux states in the ground state, and fractional ones have much higher energy. Generally, the $(L, L+n)$ energy increases further and energy levels follow each other as magnetic field and $n$ are increased.

In Fig. 19(c) we introduced the magnetic coupling, in absence of the Josephson one. This broadens the stability intervals of all vortex states-integer and fractional-as a consequence of the magnetic screening which lowers the effectively experienced field by the sample and enhances superconductivity. However, the magnetic response of the sample is generally of oblate shape (due to the symmetry of the disk), and asymmetric states are less favorable than in case (a). For that reason, pronouncedly asymmetric $(0,1)$ and $(1,2)$ states are not present in the ground state. However, higher fractional states can be found in the ground state since the ringlike arrangement of vortices and their fields in both condensates enhance each other, and also follow better the overall symmetry of the stray magnetic field. Nevertheless, Josephson coupling is still able to completely remove the fractional states from the ground state, as shown in Fig. 19(d). Arguably, at higher vorticity fractional states could appear in the ground state, since the order parameters in the two bands will become increasingly similar with increasing vorticity.

In Fig. 20 we illustrate the flux quantization in a two gap superconducting disk. As is already known, the flux in 

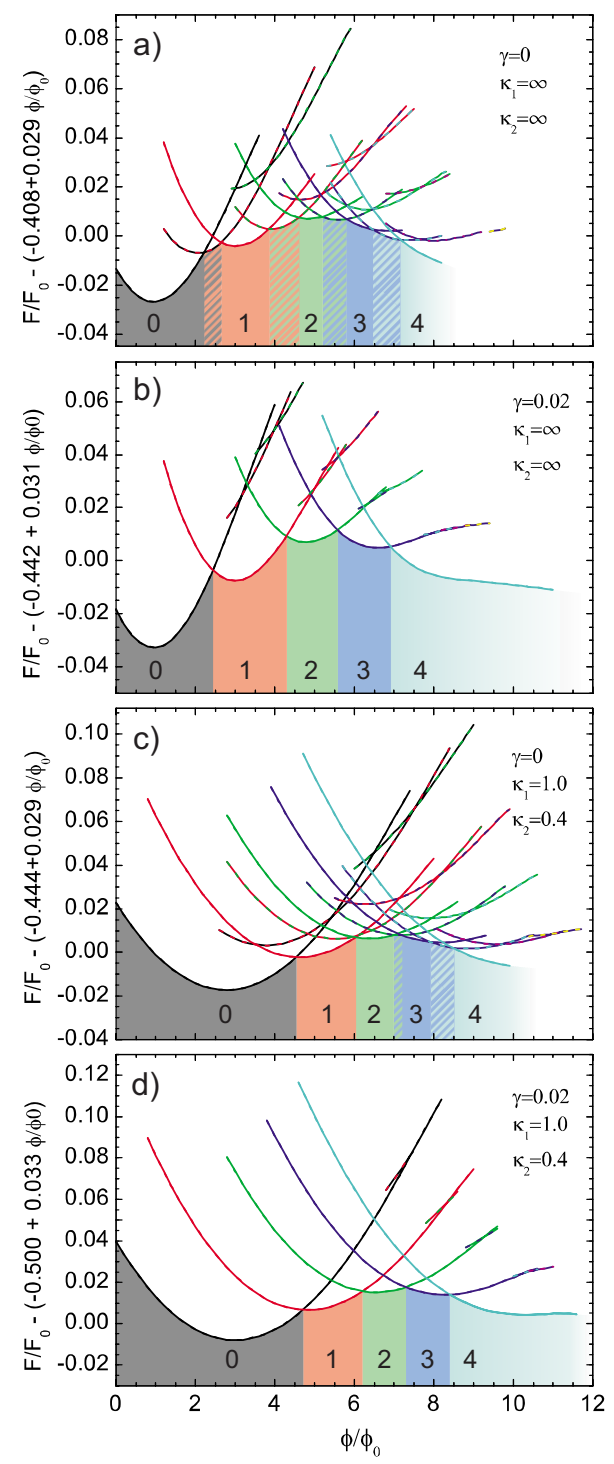

FIG. 19. (Color online) Free energy as a function of magnetic flux for found integer and fractional states with $L \leq 4$. A linear background is subtracted from all curves to enhance readability (as indicated in the labels). The colors of the curves correspond to the vorticity, and also indicate the combinations involved in the (twocolored) fractional states. The ground state is indicated by the colorcoded shaded areas below the curves. Parameters of the sample are $R_{D}=7.5 \xi_{0}, T=0.2, T_{c r}=0.5, \alpha=0.3$, and $m=1$.

single-band mesoscopic samples is not quantized, but it always is within a contour determined by the zero current. Therefore we compute the flux $\phi_{\Gamma}$ penetrating the two-band sample through a contour $\Gamma$ inside the sample on which $\vec{j}_{s}$ $=0$. The result is plotted in the presence of magnetic coupling between the bands to still have some fractional vortex states left in the ground state. By plotting the whole stability regions of the states, we noticed that the fractional flux decreases with increasing applied magnetic field (i.e., the applied flux $\phi$ ). We pinpoint this effect in addition to the findings of Ref. 19, where the authors found the decrease of flux through contour $\gamma$ with increasing temperature. The reason for the change of the fractional flux with applied field or temperature is that one of the condensates always depletes

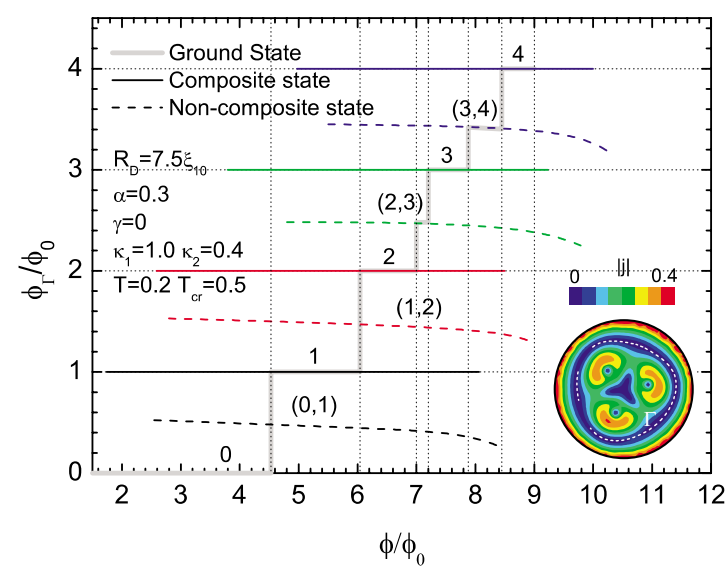

FIG. 20. (Color online) The flux quantization in a two gap superconductor bands only coupled by the magnetic field. $\phi_{\Gamma}$ is the flux measured through the contour $\Gamma$, defined as the contour where the supercurrent equals zero, as illustrated by the white dashed line in the inset. The thick solid grey line in the graph depicts the ground state as a function of applied field.

faster than the other (in the present case, the second one). At sufficiently high field or temperature, only one band superconducts, and the flux through contour $\Gamma$ changes toward its quantized level in the surviving band. In the present case, the first band is stronger, and the fractional flux decreases toward vorticity in the first band, i.e., $L=1$. If the considered state was a $(2,1)$ one, the fractional flux would increase toward $L=2$ level with increasing field or temperature.

\section{B. Magnetization curves}

We define the magnetization as: $M=\frac{1}{4 \pi V} \int \Delta H_{z} d V$, where $\Delta H$ represents the induced magnetic field. In Fig. 21 we plot the $M(H)$-loop in a $\mathrm{MgB}_{2}$ disk with radius $R_{D}=10 \xi_{0}$ and thickness $d=\xi$ at $T=0$, taking the parameters of Ref. 14, i.e., $\kappa_{1}=3.68, \kappa_{2}=0.66, \alpha=0.068$. Although the second band is conventionally type-I, the shape of the $M(H)$ loop suggests that the whole system still behaves like a type-II superconductor, i.e., there is no indication of a type 1.5 superconductivity reported by Moshchalkov et al. ${ }^{14}$ We additionally plot-

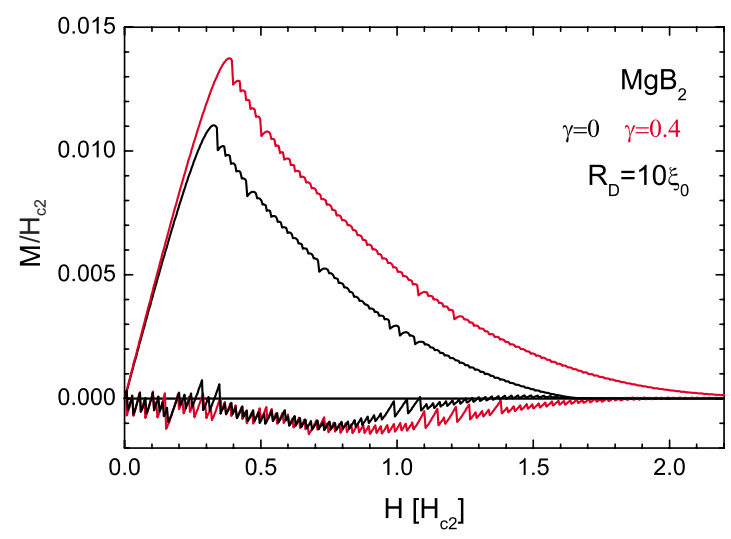

FIG. 21. (Color online) $M(H)$ loops obtained by sweeping up and down the magnetic field for a $\mathrm{MgB}_{2}$ single-crystal disk of radius $R_{D}=10 \xi_{10}$. 


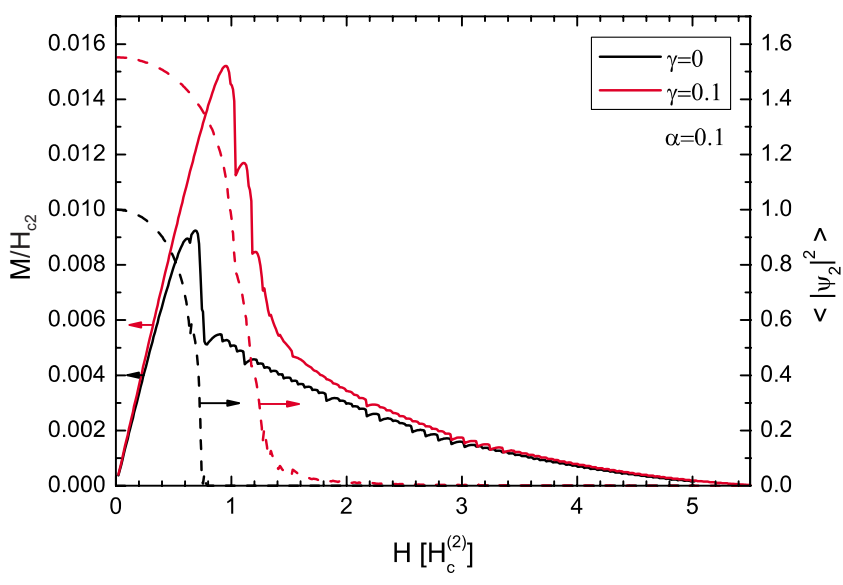

FIG. 22. (Color online) Solid curves represent magnetization as a function of applied magnetic field for $\gamma=0$ (black) and $\gamma=0.1$ (red), and parameters of the sample $\kappa_{1}=1, \kappa_{2}=0.2, \alpha=0.1, R_{D}$ $=10 \xi_{10}$, and $T=0$. Dashed curves represent the mean Cooper-pair density in the second condensate.

ted the magnetization in the absence of Josephson coupling, which also does not show any qualitative deviation of type-II behavior. The only influence of the Josephson coupling is an apparent increase of critical field and a stronger magnetic response, which is a direct consequence of currents being strengthened by coupling. However, when we take lower values of the GL parameters, for example $\kappa_{1}=1$ and $\kappa_{2}=0.2$, we find, as shown in Fig. 22 a behavior of the magnetization versus field that is neither type-II- nor type-I-like. This state is characterized by a steep drop of the magnetization at a field close to the thermodynamical critical field $H_{c}$ of the second condensate $\left(H_{c}^{(2)}\right)$. Due to finite demagnetization effects, characteristic of type-I samples, the transition is at a lower field than $H_{c}^{(2)}$. At this transition field, superconductivity ceases in the second gap, and the magnetization undergoes a steep drop. The origin of this effect is clearly visible in the figure, where also the mean Cooper-pair density in the sample is plotted-the magnetization drop coincides with the depletion of the second band. Beyond the transition field, the flux continues to enter the sample gradually, exhibiting the type-II mixed state of the first condensate, and the overall behavior of magnetization can be treated as a superposition of type-I (steep drop) and type-II (gradual decrease) behavior of the two condensates, each being of different type. The influence of the Josephson coupling is also striking, as it smoothes out the drop in the magnetization: the second condensate still depletes but at a slower rate due to the exchange of Cooper pairs with the first condensate. The slope of the decrease of the mean Cooper-pair density in the second condensate still seems to match the slope of the drop in magnetization with a remarkable accuracy, although the transition becomes less abrupt and more reminiscent of a type-I intermediate state with bundles of flux penetrating the sample.

In Figs. 23 and 24 we demonstrate the influence of the ratio of the coherence lengths, $\alpha$ on the magnetic behavior of the sample. In Eq. (15), the supercurrents due to the second condensate have a prefactor of $\alpha / \kappa_{2}^{2}$. Therefore, it is not $\kappa_{2}$ alone which determines the type of the band and an effective $\kappa_{2}^{e f f}=\kappa_{2} / \sqrt{\alpha}$ can be introduced. Essentially, this suggests that

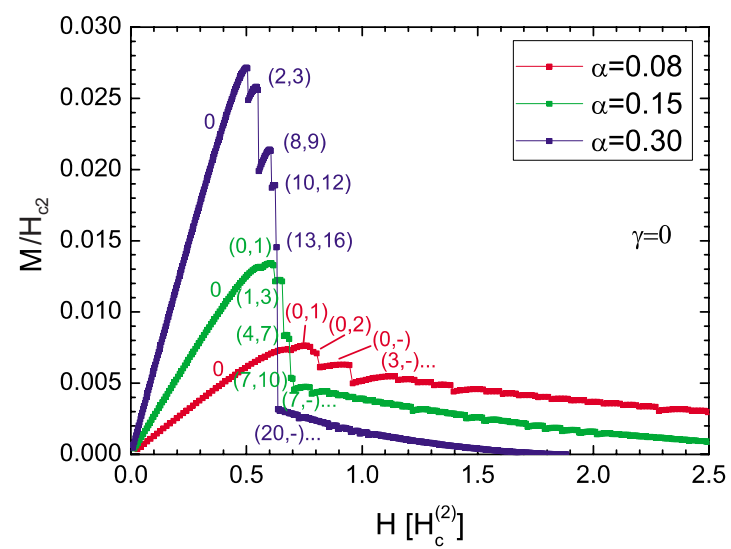

FIG. 23. (Color online) Magnetization of the sample versus the applied field for different values of the ratio between the coherence lengths in two condensates $\alpha$. Other parameters of the sample are $\gamma=0 \kappa_{1}=1, \kappa_{2}=0.2, R_{D}=10 \xi_{10}$, and $T=0$.

the self induced field should be proportional to $\alpha$, and the results in Fig. 23 are supportive of this. The level of magnetization to which the sample jumps after the magnetization drop should be independent of $\alpha$, since the second condensate is depleted there. However it turns out that the larger the magnetization is before the drop, the lower it becomes after the drop. This follows from the fact that, before the magnetization drop, the second condensate is able to provide a better screening of the magnetic field for the first condensate when $\alpha$ is larger, but when the second condensate ceases, the first condensate experiences a large difference in the felt magnetic field, which in turn allows for a larger flux penetration and thus a lower diamagnetic response of the sample.

It should be stressed here that the field at which the second condensate depletes and the magnetization drops is also influenced by $\alpha$. The smaller the coherence length of the second condensate (higher $\alpha$ ), the smaller the transition field. This difference is even more prominent in the presence of Josephson coupling. In other words, the apparent demagnetization effect in the type-I part of the magnetization curve is clearly influenced by the parameter $\alpha$.

In Fig. 25 we also show the magnetization corresponding to the sweep-down of the applied magnetic field. At the point

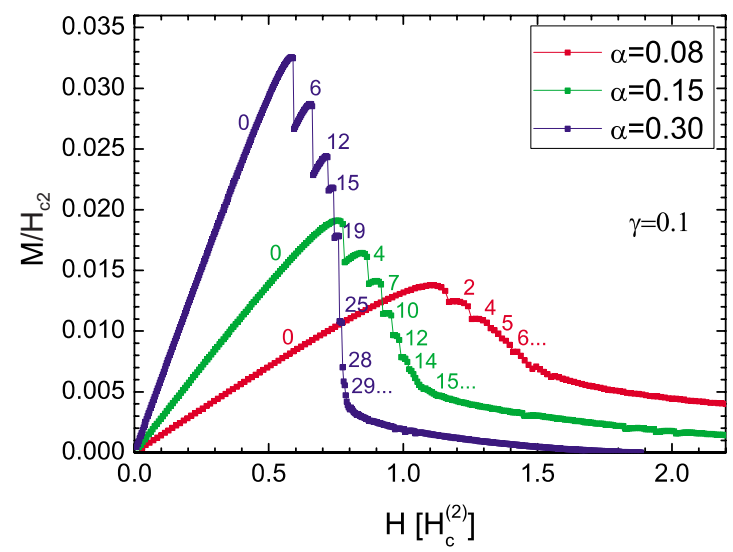

FIG. 24. (Color online) Idem as Fig. 23, but for finite Josephson coupling $\gamma=0.1$. 


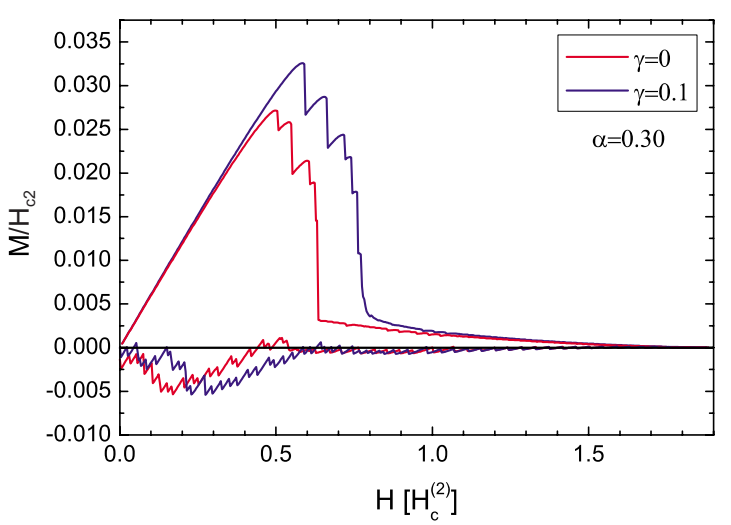

FIG. 25. (Color online) Full sweep up and down $M(H)$ loops with and without coupling. Taken parameters are $\alpha=0.3 \kappa_{1}=1, \kappa_{2}$ $=0.2, R_{D}=10 \xi_{10}$, and $T=0$.

where the second condensate revives the magnetization jumps up, since there the type-I condensate contributes to the diamagnetic signal. This jump is less abrupt when $\gamma$ is nonzero. Finally in Fig. 26, the full stability regions of the key vortex states are given for a mesoscopic type I.x superconducting disk.

\section{Type-I.x vortex states}

It is already known that vortices repel each other in type-II superconductors, form Abrikosov lattice in bulk samples, but are compressed into geometry dependent multivortex states and even giant vortex states in mesoscopic superconductors. In type-I samples however, flux penetrates the sample in the form of lamellae or tubular flux bundles. ${ }^{26}$ As we showed above, two band superconductors can show a bit of both behaviors, called type-1.5 superconductivity by Moshchalkov et al. ${ }^{14}$ While we demonstrated the type I.x behavior through the magnetization loops, they mainly discussed the vortex-vortex interaction in two-band superconductors, claiming that it should be short range repulsive and long range attractive. This of course assumes integer flux vortex states, or strong Josephson coupling in our model. However, as we have seen above, a plethora of other vortex

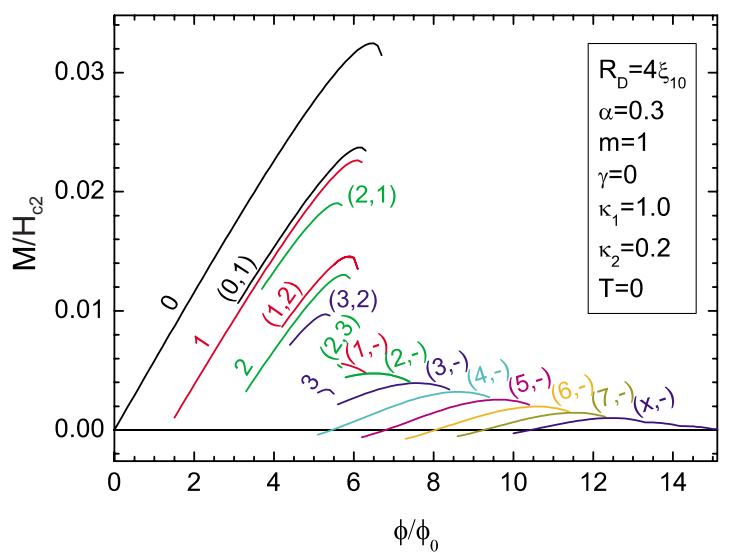

FIG. 26. (Color online) $M(H)$ for all stable vortex states in a mesoscopic type-I.x superconducting disk.

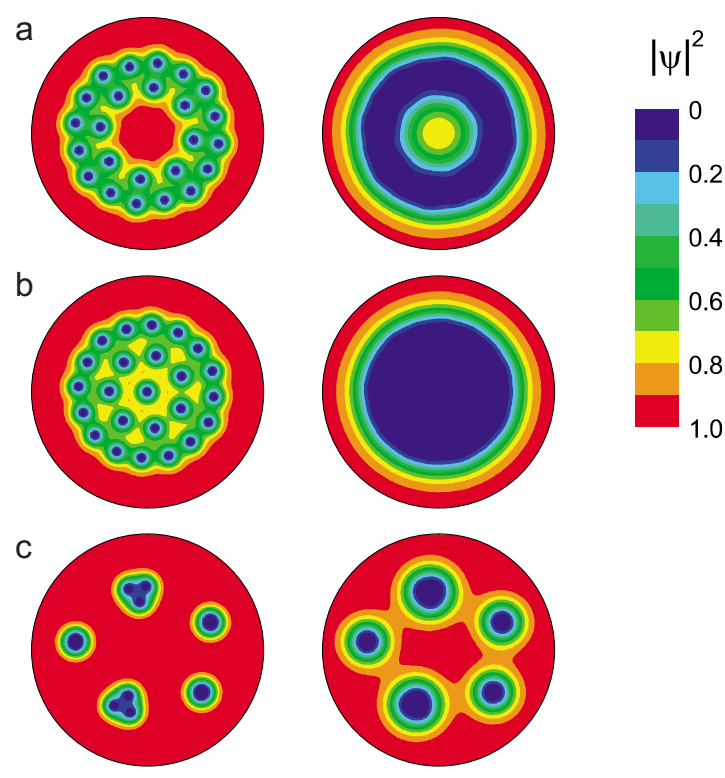

FIG. 27. (Color online) Cooper-pair density contour plots of the first (left) and second (right) condensate. $\kappa_{1}=1, \kappa_{2}=0.2, \alpha=0.3$, $R_{D}=15 \xi_{10}$. In (a) and (b) $\phi / \phi_{0}=30, L=24, \gamma=0$. In (c) $\phi / \phi_{0}=15$, $L=12, \gamma=0.02$.

states are possible, not all with integer flux. Therefore, the vortex-vortex interaction should be discussed separately within bands (intraband), and separately between them (interband). While leaving the detailed analysis for the future, we here show several prime examples of vortex states that can be found in two-band mesoscopic disks, that show typeI.x behavior (however different from Ref. 14). For example, we take the disk with radius $10 \xi_{10}$ with parameters of the condensates $\kappa_{1}=1, \kappa_{2}=0.2$, and $\alpha=0.3$. As shown in Fig. 27(a) and 27(b), at larger applied field providing 30 flux quanta through the sample, we found a $L=24$ vortex state. Due to absence of Josephson coupling, two allotropic modifications of the vortex state were found possible. In both, the vortices in type-I condensate merge into a single domain, either a ring domain (a), or a giant-bubble (b), which is typical for type-I samples. Actually they are still single vortices, but have huge overlap, mimicking a normal domain. Vortices in the type-II condensate remain separate however, but are forced by magnetic coupling to obey the symmetry of the intermediate state of the type-I condensate. They therefore form more or less a conventional multishell state under the giant vortex in (b), but are forced to make an unconventional state with dense shells within the ring domain in (a). This type-I-II competition is even more pronounced in the presence of Josephson coupling, as shown in Fig. 27(c). In the first condensate, one can see three giant $L=2$ vortices and two $L=3$ multivortex clusters. This is also the situation in the second condensate, however the multivortices are now even closer and also overlap more, mimicking perfect giant vortices. Due to the Josephson coupling, both condensates influence each other; as a consequence, the type-I intermediate state is forced to split into as many bubbles as possible, and vortices in type-II condensate must group into those bubbles. As a result, a multivortex of multivortices is formed, clearly a signature of type-I.x behavior. Similar behavior was also 
observed in Ref. 27 for a system of classical particles with short-range attractive and long-range repulsive interaction.

\section{CONCLUSIONS}

In summary, we presented a theoretical Ginzburg-Landau (GL) study of the superconducting state of two-band mesoscopic disks, where both the influence of Josephson and of magnetic coupling between the superconducting bands are discussed. In cases when screening of the magnetic field can be neglected, we found the dependence of the size of the vortex core on the strength of the Josephson coupling and showed that it generally has an influence opposite to the one of temperature. In limiting cases, our numerical findings agree well with analytic expressions available in literature. We also found a fitting function, which gives an excellent estimate of the size of the vortex core as a function of the size of the mesoscopic disk. In our further analysis of the vortex states, we focused mainly on exotic, fractional states, where two condensates comprise different number of vortices and the apparent total vorticity of the sample is fractional. We reported asymmetric vortex states following from competing interactions in the two-band mesoscopic system, and showed how some states can be manipulated by, e.g., coupling between the bands. We indicate how such states can be experimentally observed. Fractional states can even be found in the ground state, but typically far from the $\mathrm{S} / \mathrm{N}$ boundary. We give the expression for the upper critical field of a two-band mesoscopic disk as a function of temperature, which is similar to analytic estimations for bulk, however scaled to its value at zero temperature for zero coupling between the condensates.

When magnetic screening and coupling between the bands is included in the simulations, we characterized the response of the sample through the competition of the GL parameters of the two bands (with special attention to the case when one band is type-II and the other is type-I). However, we show that this is insufficient, and that Josephson coupling and the ratio of the coherence lengths in the two bands also play an important role. Although we did not find evidence for type-1.5 superconductivity in clean $\mathrm{MgB}_{2}$ disks, we did find its manifestation for a different choice of relevant parameters. The magnetization vs applied field shows a distinct jump at the field where type-I condensate ceases, and the overall shape of the curve can surely be characterized as type-I.x-like. This is also evident in the found vortex states in the latter case, which are a combination of single vortices and lamellar domains.

\section{ACKNOWLEDGMENTS}

This work was supported by the Flemish Science Foundation (FWO-Vlaanderen), the Belgian Science Policy (IAP), the ESF "Nanoscience and Engineering in Superconductivity" (NES) program, and the ESF "Arrays of Quantum Dots and Josephson Junctions" network.

\footnotetext{
*roeland.geurts@ua.ac.be

†milorad.milosevic@ua.ac.be

\#rancois.peeters@ua.ac.be

${ }^{1}$ W. Pickett, Nature (London) 418, 733 (2002).

${ }^{2}$ J. Nagamatsu, N. Nakagawa, T. Muranaka, Y. Zenitani, and J. Akimitsu, Nature (London) 410, 63 (2001).

${ }^{3}$ C. Buzea and T. Yamashita, Supercond. Sci. Technol. 14, R115 (2001).

${ }^{4}$ X. X. Xi, Rep. Prog. Phys. 71, 116501 (2008).

${ }^{5}$ L. Lyard, P. Samuely, P. Szabo, T. Klein, C. Marcenat, L. Paulius, K. H. P. Kim, C. U. Jung, H.-S. Lee, B. Kang, S. Choi, S.-I. Lee, J. Marcus, S. Blanchard, A. G. M. Jansen, U. Welp, G. Karapetrov, and W. K. Kwok, Phys. Rev. B 66, 180502(R) (2002).

${ }^{6}$ M. R. Eskildsen, M. Kugler, S. Tanaka, J. Jun, S. M. Kazakov, J. Karpinski, and Ø. Fischer, Phys. Rev. Lett. 89, 187003 (2002).

${ }^{7}$ M. Iavarone, G. Karapetrov, A. E. Koshelev, W. K. Kwok, G. W. Crabtree, D. G. Hinks, W. N. Kang, E.-M. Choi, H. J. Kim, H.-J. Kim, and S. I. Lee, Phys. Rev. Lett. 89, 187002 (2002).

${ }^{8}$ M. E. Zhitomirsky and V.-H. Dao, Phys. Rev. B 69, 054508 (2004).

${ }^{9}$ V. H. Dao and M. E. Zhitomirsky, Eur. Phys. J. B 44, 183 (2005).

${ }^{10}$ H. Doh, M. Sigrist, B. K. Cho, and S.-I. Lee, Phys. Rev. Lett. 83, 5350 (1999).

${ }^{11}$ Y. S. Yerin and A. N. Omelyanchouk, Low Temp. Phys. 33, 401 (2007).

${ }^{12}$ I. N. Askerzade, Physica C 390, 281 (2003).
}

${ }^{13}$ I. N. Askerzade, A. Gencer, and N. Güçlü, Supercond. Sci. Technol. 15, L13 (2002).

${ }^{14}$ V. Moshchalkov, M. Menghini, T. Nishio, Q. H. Chen, A. V. Silhanek, V. H. Dao, L. F. Chibotaru, N. D. Zhigadlo, and J. Karpinski, Phys. Rev. Lett. 102, 117001 (2009).

${ }^{15}$ T. Nishio, V. H. Dao, Q. Chen, L. F. Chibotaru, K. Kadowaki, and V. V. Moshchalkov, Phys. Rev. B 81, 020506(R) (2010).

${ }^{16}$ J.-P. Wang, Phys. Lett. A 374, 58 (2009).

${ }^{17}$ E. Babaev and M. Speight, Phys. Rev. B 72, 180502(R) (2005).

${ }^{18}$ E. Babaev, Phys. Rev. Lett. 89, 067001 (2002); 94, 137001 (2005); Nucl. Phys. B 686, 397 (2004); Y. Tanaka, Phys. Rev. Lett. 88, 017002 (2001); J. Phys. Soc. Jpn. 70, 2844 (2001); E. Babaev, J. Jäykkä, and M. Speight, Phys. Rev. Lett. 103, 237002 (2009).

${ }^{19}$ L. F. Chibotaru, V. H. Dao, and A. Ceulemans, EPL 78, 47001 (2007); L. F. Chibotaru and V. H. Dao, Phys. Rev. B 81, 020502(R) (2010).

${ }^{20}$ M. V. Milošević and R. Geurts, Physica C (in press), e-print arXiv:1006.1771.

${ }^{21}$ B. J. Baelus and F. M. Peeters, Phys. Rev. B 65, 104515 (2002); L. F. Chibotaru, A. Ceulemans, V. Bruyndoncx, and V. V. Moshchalkov, Nature (London) 408, 833 (2000).

${ }^{22}$ A. Kanda, B. J. Baelus, F. M. Peeters, K. Kadowaki, and Y. Ootuka, Phys. Rev. Lett. 93, 257002 (2004); D. S. Golubović, M. V. Milošević, F. M. Peeters, and V. V. Moshchalkov, Phys. Rev. B 71, 180502(R) (2005); G. R. Berdiyorov, M. V. Milošević, and F. M. Peeters, Phys. Rev. Lett. 96, 207001 
(2006); R. B. G. Kramer, A. V. Silhanek, J. Van de Vondel, B. Raes, and V. V. Moshchalkov, ibid. 103, 067007 (2009).

${ }^{23}$ G. A. Farias and F. M. Peeters, Solid State Commun. 100, 711 (1996).

${ }^{24}$ M. Eisterer, Supercond. Sci. Technol. 20, R47 (2007).

${ }^{25}$ V. V. Moshchalkov, L. Gielen, C. Strunk, R. Jonckheere, X. Qiu,
C. Van Haesendonck, and Y. Bruynseraede, Nature (London) 373, 319 (1995)

${ }^{26}$ G. R. Berdiyorov, A. D. Hernandez, and F. M. Peeters, Phys. Rev. Lett. 103, 267002 (2009).

${ }^{27}$ K. Nelissen, B. Partoens, and F. M. Peeters, Phys. Rev. E 71, 066204 (2005). 